\title{
Método Canguru segundo o Pensamento Ecossistêmico
}

\author{
Kangaroo Method according to Ecosystem Thought \\ Método Canguro según el Pensamiento Ecosistémico
}

\author{
Géssica Borges Vieira \\ ORCID: https://orcid.org/0000-0002-8556-5865 \\ Hospital Universitário Dr. Miguel Riet Corrêa Jr., Brasil \\ E-mail: borges.gessica@ hotmail.com \\ Giovana Calcagno Gomes \\ ORCID: https://orcid.org/0000-0002-2464-1537 \\ Universidade Federal do Rio Grande, Brasil \\ E-mail: giovanacalcagno@furg.br \\ Daniela Pasini \\ ORCID: https://orcid.org/0000-0002-6778-5629 \\ Hospital Universitário Dr. Miguel Riet Corrêa Jr., Brasil \\ E-mail: daniela.pasini@hotmail.com \\ Marta Regina Cezar-Vaz \\ ORCID: https://orcid.org/0000-0002-0754-7469 \\ Universidade Federal do Rio Grande, Brasil \\ E-mail: mrcezarvaz@gmail.com
}

\begin{abstract}
Resumo
Objetivo: Realizar uma aproximação entre o Método Canguru e o Pensamento Ecossistêmico. Metodologia: Estudo descritivo, qualitativo e de reflexão teórica, realizado de abril a agosto de 2021. Para fundamentação teórica acerca do Método Canguru utilizou-se o Manual Técnico do Método Canguru e em relação aos Pensamento Ecossistêmicos realizou-se uma busca nas bases de dados Literatura Internacional em Ciências da Saúde e Literatura LatinoAmericana e do Caribe em Ciências da Saúde com os descritores "Pensamento" e "Ecossistema", além da busca manual de referências bibliográficas no Google Acadêmico. O detalhamento das informações coletadas dos artigos foi realizado por meio de um instrumento que continha o título e as principais perspectivas dos Pensamentos Ecossistêmicos. Para a análise e síntese dos artigos, foi realizado um quadro sinóptico contendo as informações anteriormente descritas e os pressupostos do Método Canguru para promoção da aproximação entre as temáticas. Resultados: Por meio das ações desenvolvidas no Método Canguru foi possível constatar uma aproximação com o Pensamento Ecossistêmico por meio dos cuidados integrais; interconexão e contextualização dos serviços; visão de totalidade/unidade; interconexão, integralidade, interação, influências mútuas, cooperação e interdependência entre todos os componentes; visão holística e sistémica; e particularidades do espaço e ambiente. Considerações Finais: $\mathrm{O}$ estudo evidenciou a aproximação dos Pensamentos Ecossistêmicos no Método Canguru incorporando o cuidado ao neonato de forma integral, individualizada, humanizada, holística e de qualidade.
\end{abstract}

Palavras-chave: Método canguru; Pensamento; Ecossistema; Recém-nascido prematuro; Enfermagem neonatal.

\begin{abstract}
Objective: Bringing together the Kangaroo Method and Ecosystem Thinking. Methodology: Descriptive, qualitative and theoretical reflection study, carried out from April to August 2021. For theoretical foundation about the Kangaroo Method, the Technical Manual of the Kangaroo Method was used and, in relation to Ecosystem Thinking, a search was carried out in the databases International Literature on Health Sciences and Latin American and Caribbean Literature on Health Sciences with the descriptors "Thought" and "Ecosystem", in addition to the manual search of bibliographical references in Google Scholar. The detailing of the information collected from the articles was carried out through an instrument that contained the title and main perspectives of the Ecosystem Thoughts. For the analysis and synthesis of the articles, a synoptic table was created containing the information described above and the Kangaroo Method assumptions to promote the approximation between the themes. Results: Through the actions developed in the Kangaroo Method, it was possible to see an approximation with the Ecosystem Thinking through comprehensive care; interconnection and contextualization of services; vision of totality/unity; interconnection, integrality, interaction, mutual influences, cooperation and interdependence among all components; holistic and systemic view; and particularities of space and environment. Final Considerations: The study evidenced the approximation of Ecosystem Thoughts in the Kangaroo Method, incorporating care to the newborn in a comprehensive, individualized, humanized, holistic and quality way.
\end{abstract}

Keywords: Kangaroo-mother care method; Thinking; Ecosystem; Infant, premature; Neonatal nursing. 


\begin{abstract}
Resumen
Objetivo: Unir el método canguro y el pensamiento ecosistémico. Metodología: Estudio descriptivo, cualitativo y de reflexión teórica, realizado de abril a agosto de 2021. Para la fundamentación teórica sobre el Método Canguro se utilizó el Manual Técnico del Método Canguro y, en relación al Pensamiento Ecosistémico, se realizó una búsqueda en el bases de datos Literatura Internacional en Ciencias de la Salud y Literatura Latinoamericana y Caribeña en Ciencias de la Salud con los descriptores "Pensamiento" y "Ecosistema", además de la búsqueda manual de referencias bibliográficas en Google Scholar. El detalle de la información recolectada de los artículos se realizó a través de un instrumento que contenía el título y las principales perspectivas de los Pensamientos Ecosistémicos. Para el análisis y síntesis de los artículos se elaboró una tabla sinóptica que contiene la información descrita anteriormente y los supuestos del Método Canguro para promover la aproximación entre los temas. Resultados: A través de las acciones desarrolladas en el Método Canguro, fue posible vislumbrar un acercamiento al Pensamiento Ecosistémico a través de la atención integral; interconexión y contextualización de servicios; visión de totalidad / unidad; interconexión, integralidad, interacción, influencias mutuas, cooperación e interdependencia entre todos los componentes; visión holística y sistémica; y particularidades de espacio y medio ambiente. Consideraciones finales: El estudio evidenció la aproximación de Pensamientos Ecosistémicos en el Método Canguro, incorporando el cuidado al recién nacido de manera integral, individualizada, humanizada, holística y de calidad.
\end{abstract}

Palabras clave: Método madre-canguro; Pensamiento; Ecosistema; Recien nacido prematuro; Enfermería neonatal.

\title{
1. Introdução
}

A Declaração Universal de Direitos para o Bebê Prematuro reconhece os cuidados integrais que devem ser dirigidos ao recém-nascido prematuro (RNPT) e destaca no seu artigo XI os cuidados realizados no Método Canguru (MC) e reconhece o papel da equipe de saúde para manutenção da estrutura mínima para realização desta metodologia (Tavares, 2008/2009).

Nesta perspectiva, o MC visa garantir a humanização do cuidado neonatal, promovendo uma assistência integral e singular a cada recém-nascido $(\mathrm{RN})$, além de alicerçada na qualidade técnico-científica e nas boas práticas na área de terapia intensiva neonatal. Os cuidados propostos vão além da prematuridade ou do baixo peso ao nascer servindo de apoio ao cuidado a todo neonato que ao nascer necessite de uma internação hospitalar (Brasil, 2017).

O MC foi criado pelos neonatologistas Héctor Martinez Gómez e Edgar Rey Sanabria, em Bogotá, Colômbia, em 1979, afim de reduzir a superlotação e a mortalidade dos RNPT por meio do cuidado pele a pele e aumento do vínculo afetivo. No Brasil, esta metodologia foi introduzida na década de 90 e logo foi incorporada nas políticas de saúde na área neonatal (Gomes et al., 2021).

As práticas desenvolvidas no MC envolvem o cuidado centrado na família e a humanização da assistência neonatal, promovendo o fortalecimento do vínculo mãe-filho, o aleitamento materno, o controle da temperatura, o ganho de peso, a redução da sepse neonatal, o desenvolvimento cognitivo e motor, a estimulação sensorial, a manutenção dos sinais vitais, o empoderamento materno, a redução do período de internação e do risco de reinternação, a diminuição dos custos para saúde pública e o decréscimo na taxa de morbimortalidade infantil (Reichert et al., 2020; Cantanhede, Amorim, Oliveira, Almeida, \& Santos, 2020; Gomes et al., 2021).

A nomenclatura adotada pelo MC ocorreu devido a observação de como ocorre o nascimento de um canguru, espécie de marsupial, em que seu filhote nasce antes de completar a gestação, ou seja prematuro, e é colocado na bolsa da mãe canguru para finalizar o seu desenvolvimento e alcançar sua maturidade fisiológica (Sandes, Santos, de Menezes Gomes, Silva, \& Oliveira, 2018). Com esta metáfora é possível observar o filhote canguru como um símbolo ecológico, uma unidade ecológica, que representa o RN, além disso, seu desenvolvimento na bolsa da mãe canguru representa as interações ecossistêmicas que poderá ser caracterizado pela assistência neonatal integral e humanizada realizada nas unidades neonatais com ênfase, principalmente, no cuidado centrado na família.

O ecossistema é constituído por um grupo de elementos ou organismos interdependentes que compõem organizadamente uma unidade/totalidade, ou seja, é a interação entre elementos bióticos e abióticos ocupantes de um mesmo lugar, sendo um meio com dinamicidade, flexível e em busca de equilíbrio. O Pensamento Ecossistêmico (PE) procura 
compreender o contexto, englobando o ambiente no qual o fenômeno está acontecendo, já que a totalidade dos elementos da realidade interferem, cooperam entre si e exercem influência mutua (Forget \& Lebel, 2001; Capra \& Luisi, 2014).

É por meio das relações que se estabelecem entre os elementos constituintes de dado espaço/ambiente, que é possível perceber as influências mútuas que os elementos constituintes deste ambiente exercem com os outros, gerando mudanças, transformações, possibilitando novos caminhos/bifurcações, influenciando a dinâmica de todo o sistema (Capra \& Luisi, 2014). Assim, o ambiente da Unidade Canguru, ao propiciar a interação da mãe-filho, subsidiado pelos membros da equipe por meio do MC propicia que ambos se influenciem buscando a ordem por meio da cooperação, constituindo um todo materializado pelo fornecimento de um cuidado integral ao neonato.

Tendo em vista o exposto e reconhecendo a importância do MC como pilar para o desenvolvimento do cuidado em neonatologia, este trabalho se justifica pela necessidade de uma aproximação das ações do MC e o PE para construção de um arcabouço reflexivo das perspectivas acerca do cuidado ao neonato de forma integral, humanizada e de qualidade. Sendo assim, o objetivo do presente estudo é realizar uma aproximação entre o Método Canguru e o Pensamento Ecossistêmico.

\section{Metodologia}

Trata-se de um estudo descritivo, qualitativo e de reflexão teórica, realizado de abril a agosto de 2021. Para fundamentação teórica acerca do MC utilizou-se o Manual Técnico do Método Canguru (Brasil, 2017). Tendo como questão norteadora: Quais as perspectivas acerca do PE encontram-se disponíveis nas evidências científicas que podem ter uma aproximação com os pressupostos do MC? Buscou-se estudos nos indexadores da Biblioteca Virtual em Saúde e os artigos foram captados nas bases de dados Literatura Internacional em Ciências da Saúde (MEDLINE) e Literatura Latino-Americana e do Caribe em Ciências da Saúde (LILACS), além da busca manual de referências bibliográficas no Google Acadêmico.

Para a busca dos artigos selecionados foram utilizados os descritores com o seguinte operador booleano: "Pensamento" AND "Ecossistema”, que estão contidos nos Descritores em Ciências da Saúde (DeCS). Os critérios de inclusão estabelecidos foram: serem publicados nos últimos dez anos (de 2011 à 2021); estarem nos idiomas inglês, português ou espanhol; serem originais com textos completos disponíveis online; e que abordassem as perspectivas do PE. Foram excluídos os artigos que não comtemplavam a temática, além disso, os editoriais e as cartas ao editor.

Após a busca nas bases de dados encontramos 15 artigos, sendo nove da MEDLINE e seis da LILACS. A busca manual de referências bibliográficas acrescentou mais sete artigos, totalizando assim, 22 trabalhos, destes, depois da leitura dos títulos restaram 12 (9 excluídos por não abordarem a temática e um por duplicidade). Após a leitura dos resumos obteve-se 10 artigos que foram lidos na íntegra e utilizados neste estudo (Fluxograma 1). 
Fluxograma 1: Seleção dos artigos segundo as recomendações PRISMA (Liberati et al., 2009). Rio Grande, RS, Brasil, 2021.

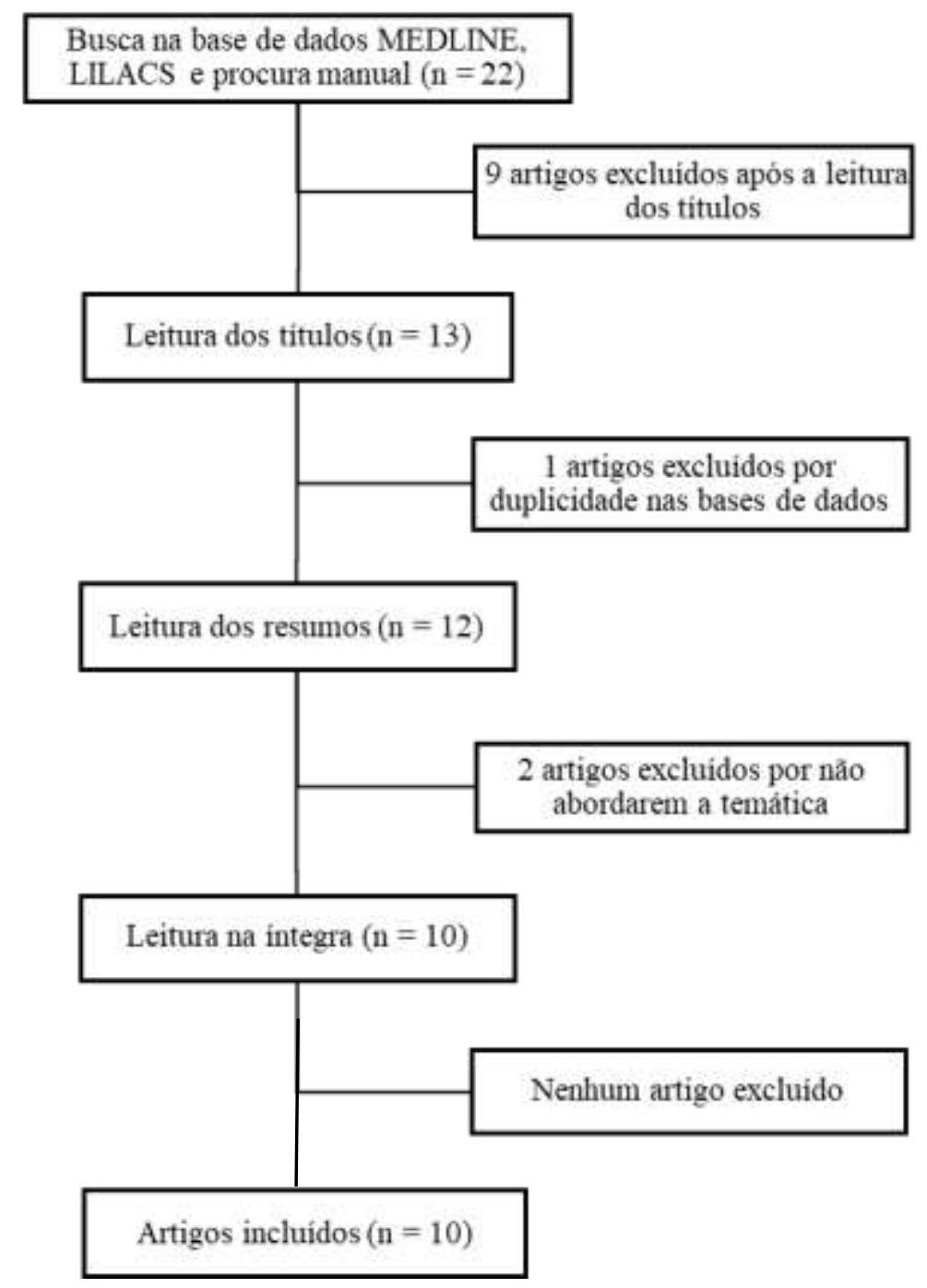

Fonte: Dados proveniente da pesquisa.

Utilizou-se um instrumento para o detalhamento das informações coletadas dos artigos, foram elas: título e as principais perspectivas acerca dos PE. Para a análise e síntese dos artigos, utilizou-se um quadro sinóptico contendo estas informações anteriormente descritas e os pressupostos do MC para promoção da aproximação entre as temáticas.

Esta reflexão teórica acerca da aproximação entre MC e o PE foi proveniente do desempenho discente da disciplina "Trabalho da Enfermagem - Saúde e Contexto Socioambiental", ministrada no curso de Doutorado em Enfermagem do Programa de Pós-Graduação em Enfermagem da Universidade Federal do Rio Grande.

\section{Resultados e Discussão}

Por meio do Quadro 1 é possível promover uma melhor visualização acerca das perspectivas do PE provenientes das evidências científicas selecionadas e a aproximação com os pressupostos do MC que serão discutidas posteriormente. 
Quadro 1: Caracterização dos artigos segundo título e perspectivas do Pensamento Ecossistêmico, além disso, exposição dos pressupostos do Método Canguru para promoção da aproximação entre as temáticas. Rio Grande, RS, Brasil, 2021.

\begin{tabular}{|c|c|c|}
\hline TÍTULO & PENSAMENTO ECOSSISTÊMICO & MÉTODO CANGURU (Brasil, 2017) \\
\hline $\begin{array}{l}\text { Obtaining tissues and organs: } \\
\text { empowering actions of nurses } \\
\text { in the light of ecosystem } \\
\text { thinking (Tolfo et al., 2021) }\end{array}$ & $\begin{array}{l}\text { Totalidade/unidade com interconexão, } \\
\text { integralidade, interação, influências mútuas, } \\
\text { cooperação e interdependência entre todos os } \\
\text { componentes do ecossistema; reações positivas } \\
\text { e/ou negativas; influências, mudanças e } \\
\text { interações decorrentes das relações e interações } \\
\text { dos componentes deste ecossistema. }\end{array}$ & $\begin{array}{l}\text { Rede de apoio para as mães; contato pele a pele; } \\
\text { estrutura mínima para permanência maternal; } \\
\text { cuidados com o recém-nascido. }\end{array}$ \\
\hline $\begin{array}{l}\text { Nurses' care for the mother- } \\
\text { baby binomial in the Stork } \\
\text { Network in the light of } \\
\text { ecosystem thinking (Thurow, } \\
\text { Sampaio, de Medeiros, Scarton, } \\
\text { \& de Siqueira, 2020) }\end{array}$ & $\begin{array}{l}\text { Cuidado integral; totalidade/unidade com } \\
\text { interconexão, integralidade, interação, } \\
\text { influências mútuas, cooperação e } \\
\text { interdependência entre todos os componentes } \\
\text { do ecossistema; reações positivas e/ou } \\
\text { negativas; consideração das particularidades do } \\
\text { espaço e ambiente onde se insere. }\end{array}$ & $\begin{array}{l}\text { Ações individuais e biopsicossocial promovendo o } \\
\text { desenvolvimento neuropsicomotor, contato pele a } \\
\text { pele, posição canguru, incentivo a participação } \\
\text { familiar e controle da dor e redução do estresse; } \\
\text { rede de apoio para as mães; contato pele a pele; } \\
\text { estrutura mínima para permanência maternal; } \\
\text { cuidados com o recém-nascido. }\end{array}$ \\
\hline $\begin{array}{l}\text { Comprehensive care from the } \\
\text { perspective of nurses: an } \\
\text { ecosystem approach (Rangel et } \\
\text { al, 2020) }\end{array}$ & Cuidado integral. & $\begin{array}{l}\text { Ações individuais e biopsicossocial promovendo o } \\
\text { desenvolvimento neuropsicomotor, contato pele a } \\
\text { pele, posição canguru, incentivo a participação } \\
\text { familiar e controle da dor e redução do estresse. }\end{array}$ \\
\hline $\begin{array}{l}\text { Adherence to nursing guidelines } \\
\text { in relation to home care of bone } \\
\text { marrow transplantees in the } \\
\text { ecosystem perscpective (Nunes } \\
\text { et al., 2020) }\end{array}$ & $\begin{array}{c}\text { Interconexão e contextualização dos serviços de } \\
\text { saúde; totalidade/unidade com interconexão, } \\
\text { integralidade, interação, influências mútuas, } \\
\text { cooperação e interdependência entre todos os } \\
\text { componentes do ecossistema; reações positivas } \\
\text { e/ou negativas. }\end{array}$ & $\begin{array}{l}\text { Desenvolvido em três etapas que englobam o } \\
\text { ambiente hospitalar e ambulatorial; rede de apoio } \\
\text { para as mães; contato pele a pele; estrutura mínima } \\
\text { para permanência maternal; cuidados com o recém- } \\
\text { nascido. }\end{array}$ \\
\hline $\begin{array}{l}\text { Participação Social Na Saúde e } \\
\text { o Papel da Enfermagem: } \\
\text { Aplicação do Modelo Ecológico } \\
\text { (Ferreira et al., 2019) }\end{array}$ & $\begin{array}{l}\text { Interação entre os componentes do ecossistema; } \\
\text { adaptações ambientais. }\end{array}$ & $\begin{array}{l}\text { Rede de apoio para as mães; intervenções } \\
\text { biopsicossocial com ambiência que abrange o } \\
\text { cuidado ao RN e sua família. }\end{array}$ \\
\hline $\begin{array}{l}\text { Educação em saúde provida } \\
\text { pelo enfermeiro ao cuidador à } \\
\text { luz do pensamento } \\
\text { ecossistêmico (de Paula et al, } \\
\text { 2017) }\end{array}$ & $\begin{array}{c}\text { Totalidade/unidade com interconexão, } \\
\text { integralidade, interação, influências mútuas, } \\
\text { cooperação e interdependência entre todos os } \\
\text { componentes do ecossistema; reações positivas } \\
\text { e/ou negativas. }\end{array}$ & Rede de apoio para as mães. \\
\hline $\begin{array}{l}\text { The evolution of health as na } \\
\text { ecological concept (Mallee, } \\
\text { 2017) }\end{array}$ & $\begin{array}{l}\text { Visão holística e perspectiva sistémica, além } \\
\text { de, explorar como a saúde do ser humano e o } \\
\text { ecossistema estão ligados. }\end{array}$ & $\begin{array}{l}\text { Contato pele a pele; estrutura mínima para } \\
\text { permanência maternal; cuidados com o recém- } \\
\text { nascido. }\end{array}$ \\
\hline $\begin{array}{l}\text { Comprehensiveness and } \\
\text { humanization of nursing care } \\
\text { management in the Intensive } \\
\text { Care Unit (Medeiros et al., } \\
\text { 2016) }\end{array}$ & $\begin{array}{c}\text { Interconexão e contextualização dos serviços de } \\
\text { saúde; visão de unidade/totalidade, organização } \\
\text { e funcionamento dos serviços de saúde com } \\
\text { ações de interconexão e interações dinâmicas, } \\
\text { complexas e mútuas, além da consideração das } \\
\text { particularidades do espaço e ambiente onde se } \\
\text { insere. }\end{array}$ & $\begin{array}{l}\text { Contato pele a pele; estrutura mínima para } \\
\text { permanência maternal; cuidados com o recém- } \\
\text { nascido. }\end{array}$ \\
\hline $\begin{array}{l}\text { Interconexão entre a história da } \\
\text { graduação em enfermagem no } \\
\text { Brasil e o pensamento } \\
\text { ecossistêmico (Severo \& } \\
\text { Siqueira, 2013) }\end{array}$ & $\begin{array}{c}\text { Cooperação, parceria, inter-relação e } \\
\text { interdependência são capazes de exercer } \\
\text { influência mútua e, assim, construir um produto } \\
\text { com capacidade de transformar o meio no qual } \\
\text { se insere. }\end{array}$ & $\begin{array}{l}\text { Rede de apoio para as mães; contato pele a pele; } \\
\text { estrutura mínima para permanência maternal; } \\
\text { cuidados com o recém-nascido. }\end{array}$ \\
\hline $\begin{array}{l}\text { Complexity of nursing care of } \\
\text { the elderly people: reflections } \\
\text { on the health of the ecosystem } \\
\text { approach (Hammerschmidt, } \\
\text { Santos, Erdmann, Caldas, \& } \\
\text { Lunardi, 2013) }\end{array}$ & $\begin{array}{c}\text { Interdependência, interatividade, mudança e } \\
\text { multidimensionalidade. }\end{array}$ & $\begin{array}{l}\text { Rede de apoio para as mães; intervenções } \\
\text { biopsicossocial com ambiência que abrange o } \\
\text { cuidado ao RN e sua família. }\end{array}$ \\
\hline
\end{tabular}


O MC objetiva reduzir os malefícios advindos de um nascimento inesperado, sendo desenvolvido por meio da ação individual e biopsicossocial a partir do cuidado particular promovido ao RN enfermo e/ou RNPT e/ou de baixo peso ao nascer e sua família. Destaca-se a adoção de cuidados individualizados, contingentes, integrais e humanizados no manuseio ao neonato visando o desenvolvimento neuropsicomotor, além do contato pele a pele, da posição canguru, do incentivo a participação familiar, das ações de controle da dor e redução do estresse (Brasil, 2017; Reichert et al., 2020).

O cuidado integral da saúde que subsidia o MC faz parte da estrutura das ações e serviços desenvolvidos segundo as perspectivas teóricas e metodológicas do PE (Thurow, Sampaio, de Medeiros, Scarton, \& de Siqueira, 2020), assim o cuidado integral é promovido a partir dos elementos do ecossistema e das relações provenientes, indo além da perspectiva biológica ao abranger o sociocultural, ambiental, espiritual e as interações provenientes estabelecidas (Rangel et al., 2020).

O MC é desenvolvido em três etapas, a primeira, inicia-se no pré-natal da gestante que precisa de cuidados especiais, durante o trabalho de parto de alto risco e durante a internação do RN na Unidade Intensiva Neonatal ou na Unidade de Cuidado Intermediário Neonatal Convencional; a segunda, o bebê permanece continuamente com sua mãe, na posição canguru, na Unidade de Cuidado Intermediário Neonatal Canguru; a terceira, é no domicilio, com seguimento ambulatorial (Follow-up) e da Unidade Básica de Saúde. Importante ressaltar o incentivo da presença e participação do pai durante todo o processo, além dos irmãos e demais familiares (Brasil, 2017; Reichert et al., 2020; Alves et al., 2020). Sendo assim, a assistência desenvolvida no MC envolve o ambiente hospitalar e ambulatorial, estes serviços devem estar interconectados e contextualizados para desenvolver um cuidado integral e de qualidade, elementos estes do PE (Medeiros et al., 2016; Nunes et al., 2020).

Além disso, cabe ressaltar a importância de uma rede de apoio para as mães neste período que irá fazer parte familiares, amigos, outras mães-canguru, profissionais de saúde tanto no âmbito hospitalar quanto na atenção primária (Brasil, 2017; Reichert et al., 2020). O apoio do profissional de saúde é primordial durante todas as etapas do MC, sendo necessário a explicação e repetição quantas vezes forem necessárias das peculiaridades acerca do cuidado a este RN até a que ocorra segurança da família na realização destes cuidados (Cantanhede, Amorim, Oliveira, Almeida, \& Santos, 2020; Gomes et al., 2021).

Neste contexto, destaca-se os princípios do PE que formam uma totalidade/unidade com a interconexão, integralidade, interação, influências mútuas, cooperação e interdependência entre todos os componentes deste ecossistema (mães, familiares, amigos, outras mães-canguru, profissionais de saúde), além disso, é possível verificar que estes componentes se influencias e podem geram reações positivas e/ou negativas (Severo \& Siqueira, 2013; Hammerschmidt, Santos, Erdmann, Caldas, \& Lunardi, 2013; de Paula et al., 2017; Ferreira et al., 2019; Thurow, Sampaio, de Medeiros, Scarton, \& de Siqueira, 2020; Tolfo et al., 2021; Nunes et al., 2020).

Desenvolvido por meio de uma assistência qualificada e humanizada o MC agrupa intervenções biopsicossocial com ambiência que abrange o cuidado ao RN e sua família. A característica principal do método é o contato pele a pele que se inicia precocemente por meio de um toque e evoluindo até a posição canguru. O método ainda visa garantir no ambiente hospitalar uma estrutura mínima para permanência da mãe, como local para dormir, banheiro, alimentação e atividades manuais. O MC ainda destaca os cuidados em algumas alterações clínicas neonatais como na apneia, no refluxo gastroesofágico, na displasia broncopulmonar, na anemia e no controle térmico, além de abordar os aspectos nutricionais, da amamentação e cuidados com a pele. Abrange ainda os cuidados voltados para preservação do sono, posturais, redução de estímulos sonoros e visuais, manuseio mínimo, agrupamento dos cuidados, banho, pesagem e troca de fralda (Brasil, 2017).

Com isso é possível observar o cuidado neonatal de forma holística e humana alicerçado nos PE em que a saúde do ecossistema incorpora dimensões éticas, possui uma visão holística e perspectiva sistémica, além de, explorar como a saúde do ser humano e o ecossistema estão ligados (Mallee, 2017). Além disso, destaca-se na Abordagem Ecossistêmica a visão de 
unidade/totalidade, organização e funcionamento dos serviços de saúde com ações de interconexão e interações dinâmicas, complexas e mútuas, além da consideração das particularidades do espaço e ambiente onde se insere (Severo \& Siqueira, 2013; Medeiros et al., 2016; Thurow, Sampaio, de Medeiros, Scarton, \& de Siqueira, 2020; Nunes et al., 2020) e a adaptação ambiental (ambiência) (Hammerschmidt, Santos, Erdmann, Caldas, \& Lunardi, 2013; Ferreira et al., 2019). Deste modo, é possível observar as influencias, mudanças e interações decorrentes da relação e interação dos componentes deste ecossistema (Tolfo et al., 2021).

Das limitações desta reflexão, ressaltamos a ausência de estudo que contemplem o MC e o PE, em que a aproximação entre estas temáticas ocorreu por meio da inter-relação com outros campos de atuação, demonstrando assim uma lacuna de conhecimento a ser trabalhada. Dentre as contribuições deste estudo, destacamos o arcabouço reflexivo gerado acerca das ações do MC e a Abordagem Ecossistêmica como forma de promover o cuidado ao neonato, fortalecendo, assim, o conhecimento na área de neonatologia.

\section{Considerações Finais}

A incorporação do PE no campo da neonatologia ganhou destaque com o MC ao adotar como símbolo ecológico o canguru, destacando o nascimento prematuro e os cuidados individualizados necessários a este neonato que ainda encontra-se em desenvolvimento, assim como observado no bebê canguru que nasce prematuramente e é colocado na bolsa da mãe canguru para completar o seu desenvolvimento.

O estudo evidenciou a aproximação dos PE no MC incorporando o cuidado ao neonato de forma integral, individualizada, humanizada, holística e de qualidade. Além da interconexão e interação dos serviços de saúde da atenção hospitalar e ambulatorial na assistência ao neonato e principalmente na continuidade do cuidado prestado.

Além disso, o cuidado dispensado ao RNPT e sua família percebido através da humanização da assistência reflete o entendimento de que a natureza socioambiental de uma unidade neonatal não constitui simplesmente o conjunto de tecnologias existentes, influenciando o meio social ali presente, mas constitui uma teia de relações complexas e em constante influência mútua. Os seres que interagem deixam de ser apenas objetos, eles se fazem sujeitos, sempre relacionados e interconectados, formando um complexo sistema ecossocial.

Contudo, projeta-se a necessidade de mais estudos científicos que contribuam para o desenvolvimento de um possível alicerce de conhecimento a respeito da prematuridade e a sua interconexão com o PE. Acredita-se, que após esta reflexão seja possível estimular o desenvolvimento de novas perspectivas a respeito do ambiente ecossistêmico e a sua relação com a prematuridade, a rede de apoio social que surge neste contexto e cuidado integral em saúde, através da emergência de novas formas de perceber e entender a complexidade do cuidado de enfermagem em neonatologia.

\section{Referências}

Alves, F. N., Azevedo, V. M. G. D. O., Moura, M. R. S, Ferreira, D. M. D. L. M., Araújo, C. G. A., Mendes-Rodrigues, C., \& Wolkers, P. C. B. (2020). Impacto do método canguru sobre o aleitamento materno de recém-nascidos pré-termo no Brasil: uma revisão integrativa. Ciência \& Saúde Coletiva, 25:45094520 .

Brasil. Ministério da Saúde. Secretaria de Atenção à Saúde. Departamento de Ações Programáticas Estratégicas. Atenção humanizada ao recém-nascido: Método Canguru: manual técnico / Ministério da Saúde, Secretaria de Atenção à Saúde, Departamento de Ações Programáticas Estratégicas. (3a ed.), Ministério da Saúde, 2017, 340 p.: il.

Cantanhede, E. S., Amorim, F. C. M., Oliveira, A. D. D. S., Almeida, C. A. P. L., \& Santos, S. M. D. (2020). Mothers' experiences in caring for premature newborn in the Kangaroo method. Cogitare Enfermagem, 25.

Capra, F., \& Luisi, P. L. (2014). A visão sistêmica da vida: uma concepção unificada e suas implicações filosóficas, políticas, sociais e econômicas. São Paulo: Cultrix. 
de Paula, S. F., De Siqueira, H. C. H., Medeiros, A. C., Rangel, R. F., Rodrigues, S. T., \& Pedoroso, V. S. M. (2017). Educação em saúde provida pelo enfermeiro ao cuidador à luz do pensamento ecossistêmico. Research, Society and Development, 9(4), e63942854-e63942854.

Ferreira, A. G., Carvalho, D. P. C., Barlem, E. L. D., Rocha, L. P., Silva, M. R. S. D., \& Vaz, M. R. C. (2019). Participação social na saúde e o papel da enfermagem: aplicação do modelo ecológico. Rev Fund Care Online, 11(5), 1360-7.

Forget, G., \& Lebel, J. (2001). An Ecosystem Approach to Human Health. International Journal of Occupational and Environmental Health, 7(2).

Gomes, M. P., Saráty, S. B., Pereira, A. A., Parente, A. T., Santana, M. E. D., Cruz, M. D. N. D. S, \& Figueira, A. D. M. (2021). Mothers' knowledge of premature newborn care and application of Kangaroo Mother Care at home. Revista Brasileira de Enfermagem, 74.

Hammerschmidt, K. S. A., Santos, S. S. C., Erdmann, A. L., Caldas, C. P., \& Lunardi, V. L. (2013). Complexity of nursing care of the elderly people: reflections on the health of the ecosystem approach. Cienc Cuid Saude, 12(1), 191-196.

Liberati, A., Altman, D. G., Tetzlaff, J., Mulrow, C., Gøtzsche, P., Ioannidis JPA, Clarke, M., Devereaux, J. K, \& Moher, D. (2009). The PRISMA statement for reporting systematic reviews and meta-analyses of studies that evaluate health care interventions: explanation and elaboration. PLoS Med, 6(7):e1000100.

Mallee H. (2017). The evolution of health as an ecological concept. Current Opinion in Environmental Sustainability, (25):28-32.

Medeiros, A. C. D., Siqueira, H. C. H. D., Zamberlan, C., Cecagno, D., Nunes, S. D. S., \& Thurow, M. R. B. (2016). Comprehensiveness and humanization of nursing care management in the Intensive Care Unit. Revista da Escola de Enfermagem da USP, 50(5):816-822.

Nunes, S. D. S., Montesinos, M. J. L., Pedroso, V. S. M., Tolfo, F., Bick, M. A., \& Siqueira, H. C. H. D. (2020). Adherence to nursing guidelines in relation to home care of bone marrow transplantees in the ecosystem perscpective. Texto \& Contexto-Enfermagem, 29.

Rangel, R. F., Paula, S. F. D., Zamberlan, C., Backes, D. S., Medeiros, A. C. D., \& Siqueira, H. C. H. D. (2020). Comprehensive care from the perspective of nurses: an ecosystem approach. Revista Brasileira de Enfermagem, 73.

Reichert, A. P. D. S., Soares, A. R., Bezerra, I. C. D. S., Dias, T. K. C., Guedes, A. T. A., \& Vieira, D. D. S. (2020). Maternal experience with the kangaroo method at home. Revista Mineira de Enfermagem, 24, 1-8.

Sandes, J. L., Santos, D. L., de Menezes Gomes, J., Silva, G. S., \& Oliveira, C. M. (2019). Atuação do fisioterapeuta e a resposta do recém-nascido ao Método Canguru: estudo documental. Revista Saúde-UNG-Ser, 12(3/4), 14-22.

Severo, D. F., \& Siqueira, H. C. H. D. (2013). Interconexão entre a história da graduação em enfermagem no Brasil e o pensamento ecossistêmico. Revista Brasileira de Enfermagem, 66, 278-281.

Tavares, L. A. M. (2008/2009). Uma declaração universal de direitos para o bebê prematuro. Rio de Janeiro: Diagraphic.

Thurow, M. R. B., Sampaio, A. D., de Medeiros, A. C., Scarton, J., \& de Siqueira, H. C. H. (2020). Nurses' care for the mother-baby binomial in the Stork Network in the light of ecosystem thinking. Research, Society and Development, 9(10): e9099109221-e9099109221.

Tolfo, F., Siqueira, H. C. H. D., Scarton, J., Cezar-Vaz, M. R., Santos, J. L. G. D., Rodrigues, S. T., \& Pedroso, V. S. M. (2021). Obtaining tissues and organs: empowering actions of nurses in the light of ecosystem thinking. Revista Brasileira de Enfermagem, 74. 